\title{
CCD Photometry of Variable Stars in the Galactic Open Cluster NGC 7419
}

\author{
Z. Kołaczkowski, A. Pigulski, G. Kopacki \\ Wroctaw University Observatory, Kopernika 11, 51-622 Wroctaw, \\ Poland
}

\begin{abstract}
We present results of a CCD variability search in the field of the young open cluster NGC 7419. This cluster contains a large number of Be stars and almost all of them turned out to be variable. They show mostly irregular variations on different time-scales with ranges up to 0.4 mag in the $I_{C}$ band. The most interesting result is the discovery of shortperiod variability in some of the Be stars.
\end{abstract}

\section{The cluster}

NGC $7419(\mathrm{C} 2252+605)$ is a young open cluster in Cepheus. It contains five $\mathrm{M}$-type supergiants. One of them is MY Cep, an Lb or SRc-type variable of MK type M7.5 I. NGC 7419 is highly reddened. Its $E(B-V)$ amounts to about $2 \mathrm{mag}$ and varies across the cluster (Beauchamp et al., 1994). The cluster age was estimated by Bhatt et al. (1993) to be smaller than $40 \mathrm{Myr}$, whereas Beauchamp et al. (1994) derived a value of about 14 Myr. Recently (Pigulski \& Kopacki, 2000), we made a photometric search for $\mathrm{H} \alpha$ emission objects in this cluster; we discovered 17 new Be stars. The fraction of Be stars in NGC 7419 amounts to $36 \%$, which makes the cluster one of the most abundant in Be stars.

\section{Observations}

NGC 7419 was searched for short-period B-type pulsators. The small angular diameter, young age, high density and low contamination by foreground and background stars make it a very promising object for such kind of study. Photometric observations were made at Bialków Observatory of the Wroclaw University with a $0.6-\mathrm{m}$ reflecting telescope equipped with a CCD camera $\left(4^{\prime} \times 6^{\prime}\right.$ field of view). The observations were carried out in two seasons, 1999 and 2000.

To minimize the effect of interstellar extinction, most CCD frames, i.e. 1045 , were taken through the $I_{\mathrm{C}}$ filter. Additionally, we obtained 55 and 91 frames in $R_{\mathrm{C}}$ and $V$ bands, respectively. All frames were reduced using the point spread function fitting algorithm of the DAOPHOT II package (Stetson, 1987). We identified 536 stars in the observed field. 


\section{The main result: Be stars with short-period variability}

All stars with average photometric error smaller than 0.2 mag were checked for variability and 29 were found to be variable. All of them are members of the cluster. Out of the 31 Be stars we had in our search field, 26 turned out to be variable. Most of them show complicated, irregular light changes. However, in three cases we detected short-period variations. These are BMD 692, BMD 551 and BMD 451, where BMD indicates that the numbers are those of Beauchamp et al. (1994).

The star BMD 692 shows strong emission in $\mathrm{H} \alpha$ and long-term irregular light variations with a range of about 0.12 mag. Between HJD 2451716 and HJD 2451778 , however, we detected variations with a single period of $0.3559 \mathrm{~d}$ and a semi-amplitude of $14 \mathrm{mmag}$. These variations are most likely due to rotation, so that the star can be classified as a $\lambda$ Eri-type variable. Since double or even triple waves have been observed in some of these stars, the true rotation period is uncertain at this stage.

Short-period variations were also found in two other Be stars, BMD 551 and BMD 451. The differential photometry of BMD 551 yields a single period of $0.26188 \mathrm{~d}$, probably too short to be explained by rotation. Since the star lies on the cluster main sequence in a position corresponding to spectral type B2, these variations can be interpreted as pulsations of the $\beta$ Cephei type.

The other star, BMD 451, clearly shows two periodicities. Unfortunately, the daily aliases are very strong; the most probable periods are: $P_{1}=0.3803 \mathrm{~d}$ and $P_{2}=0.2766 \mathrm{~d}$. It is possible that these periodicities are both due to $\beta$ Cephei-type pulsations. If confirmed, the two stars would join $27 \mathrm{CMa}$ (Balona \& Rozowsky, 1991) as Be stars showing $\beta$ Cephei-type pulsations. 19.

Acknowledgments. This work was supported by KBN grant 2P03D 006

\section{References}

Balona, L.A. \& Rozowsky, J. 1991, MNRAS, 251, 66

Beauchamp, A., Moffat, A.F.J., \& Drissen, L. 1994, ApJS, 93, 187

Bhatt, B.C., Pandey, A.K., Mohan, V., Mahra, H.S., \& Paliwal, D.C., 1993, Bull. Astron. Soc. India, 21, 33

Pigulski, A. \& Kopacki, G., 2000, A\&AS, 146, 465

Stetson, P.B. 1987, PASP, 99, 191 